\title{
Management of Electrical Storm: The Answer Lies outside the Heart \\ Venkatesh $\mathrm{K}^{*}$, Acevedo J, Santos MD, Kim N, Catanzaro J and Hsu SS
}

University of Florida Health, Jacksonville, Florida, USA

${ }^{*}$ Corresponding author: Karthik Venkatesh, Department of Medicine, Division of Cardiology, University of Florida Health, Jacksonville, Florida, 32209, USA, Tel: 904-383-1011; E-mail: dr.vkarthik@gmail.com

Received date: May 26, 2017; Accepted date: June 19, 2017; Published date: June 26, 2017

Copyright: (c) 2017 Venkatesh K, et al. This is an open-access article distributed under the terms of the Creative Commons Attribution License, which permits unrestricted use, distribution, and reproduction in any medium, provided the original author and source are credited.

\section{Introduction}

Ventricular Arrhythmias (VA) in the setting of advanced structural heart disease are common. Implantable Cardiac Defibrillators (ICD) is used in patients at risk for VA or survivors of aborted Sudden Cardiac Death (SCD). However, in up to $20 \%$ of these patients, there is a significant incidence of electrical storm (described as 3 or more episodes of VA within a 24-h period) that results in several ICD shocks and increases mortality [1]. Management of this particular set of patients poses a clinical challenge which requires a multi-modality approach, one of them involving modulation of sympathetic nervous system [2]. We describe a similar case of a patient with refractory VA who underwent a multi-disciplinary approach resulting in good outcome.

\section{Case Report}

A 72-year-old gentleman with a history of hypertension, diabetes mellitus, dilated cardiomyopathy and interventricular conduction delay underwent implantation of a biventricular ICD in 2007 for primary prevention of SCD. In 2010 he had recurrent sustained monomorphic Ventricular Tachycardia (VT) and ICD shocks and underwent endocardial catheter ablation (substrate modification) and amiodarone initiation. Shortly thereafter he had recurrence of VT and underwent a second catheter ablation (endocardial and epicardial substrate based ablation) with change in anti-arrhythmic therapy to sotalol and mexiletine. In 2014 he had another round of VT and ICD shocks and this time he was switched over to dofetilide and phenytoin with VT suppression over the next 2 years.

In March 2017 while on vacation away from home, he presented to a local community hospital with recurrent ICD shocks, this time in Electrical Storm (ES), described as 3 or more shocks for VA within 24 h. He was initiated on IV Amiodarone +IV Lidocaine with continued VT and ICD shocks. Emergent coronary angiogram showed non obstructive coronary disease. Echocardiogram showed severe left ventricle systolic dysfunction with an ejection fraction of $10-15 \%$. Unable to suppress VT, he underwent deep sedation (Richmond Agitation Sedation Scale/RASS of -4) with propofol and subsequent endotracheal intubation/mechanical ventilation. This resulted in VT suppression and he was transferred to a tertiary care center for further management.

At the tertiary care center any attempts at weaning off propofol (even RASS of -2) would result in recurrent VT and ICD shocks, while he remained on Metoprolol, IV amiodarone and IV lidocaine. Reversible causes such as electrolyte abnormalities, left ventricular pacing from region of scar, arrhythmogenic drugs etc. were absent. After two days of VT suppression with deep sedation with propofol, we attempted endocardial catheter ablation for substrate modification of VT. However, catheter stimulation and radiofrequency ablation in the left ventricle resulted in significant amount of VT and Ventricular Fibrillation (VF) resulting in extreme hemodynamic instability (even with the use of a percutaneous left ventricular assist device/Impella $\mathrm{CP}$ ) (Figure 1). Hence the procedure was aborted and decision was made to attempt sympathetic nervous system modulation.

Patient was subsequently transferred to another tertiary care center where he underwent bilateral percutaneous cervical stellate ganglion (sympathetic) block initially using bupivacaine with reasonable success. Later on he underwent bilateral cervical sympathetic denervation surgically resulting in complete VT suppression over the next 7 days. He was subsequently weaned off propofol and IV lidocaine and discharged home on oral amiodarone with no neurological deficits.

\section{Discussion}

Propofol is generally used as an intravenous general anesthetic or as a sedative in mechanically ventilated patients. This effect is due to the decrease in NMDA-mediated excitatory neurotransmission. Propofol has also been used as an anticonvulsant. It is used typically during status epilepticus when conventional agents fail. This effect may be due to enhanced inhibitory synaptic transmission from GABA receptor agonism or glutamate receptor inhibition. Additionally, propofol has been used for its anti-emetic properties by decreasing serotonin and 5 hydroxyindoleacetic acid concentration.

Propofol has a large volume of distribution secondary to the high lipophilicity which results in rapid onset and offset of effects. Propofol is metabolized in liver by CYP2B6 however also has extra-hepatic metabolism. Since propofol is made of a lipid emulsion, patients need to be assessed if they are allergic to egg, soy, and peanuts. Also use in caution in patients with hypertriglyceridemia secondary to the lipid emulsion. Additionally, apnea and hypotension are relatively common in patients with propofol use.

In addition to the above properties, propofol has anti-arrhythmic effects. In the literature there are case reports of propofol being used to treat atrial arrhythmias and ventricular tachycardia [3-5]. The possible antiarrhythmic mechanisms of propofol include ion channel inhibition, uneven suppression of the autonomic nervous system, and protection of gap junctions during ischemia [6].

Of particular interest is the effect of propofol on the sympathetic nervous system. There is existing evidence on the role of cardiac sympathetic nervous system on ventricular arrhythmias, even in the absence of structural heart disease [7]. Nademanee demonstrated the effect of sympathetic blockade on VT suppression by using either betablockers or sympathetic blockade with percutaneous stellate ganglion block using xylocaine [8]. Early multicenter case series of surgical left cardiac sympathetic denervation in patients with incessant drug refractory VA initially showed mixed short term outcomes with 
Citation: Venkatesh K, Acevedo J, Santos MD, Kim N, Catanzaro J, et al. (2017) Management of Electrical Storm: The Answer Lies outside the Heart. Cardiovasc Pharm Open Access 6: 213. doi:10.4172/2329-6607.1000213

Page 2 of 3

regards to VT suppression [9]. A very limited experience with randomized spinal cord stimulation (of the cervical sympathetic system) showed promising results pending further investigation [10]. Bilateral cardiac sympathetic denervation has been performed in similar patients with refractory VT with favorable outcomes (improved ICD shock free survival). This came at the expense of certain side effects such as changes in sweating pattern, ptosis, skin sensitivity etc. among a small group of patients [11].

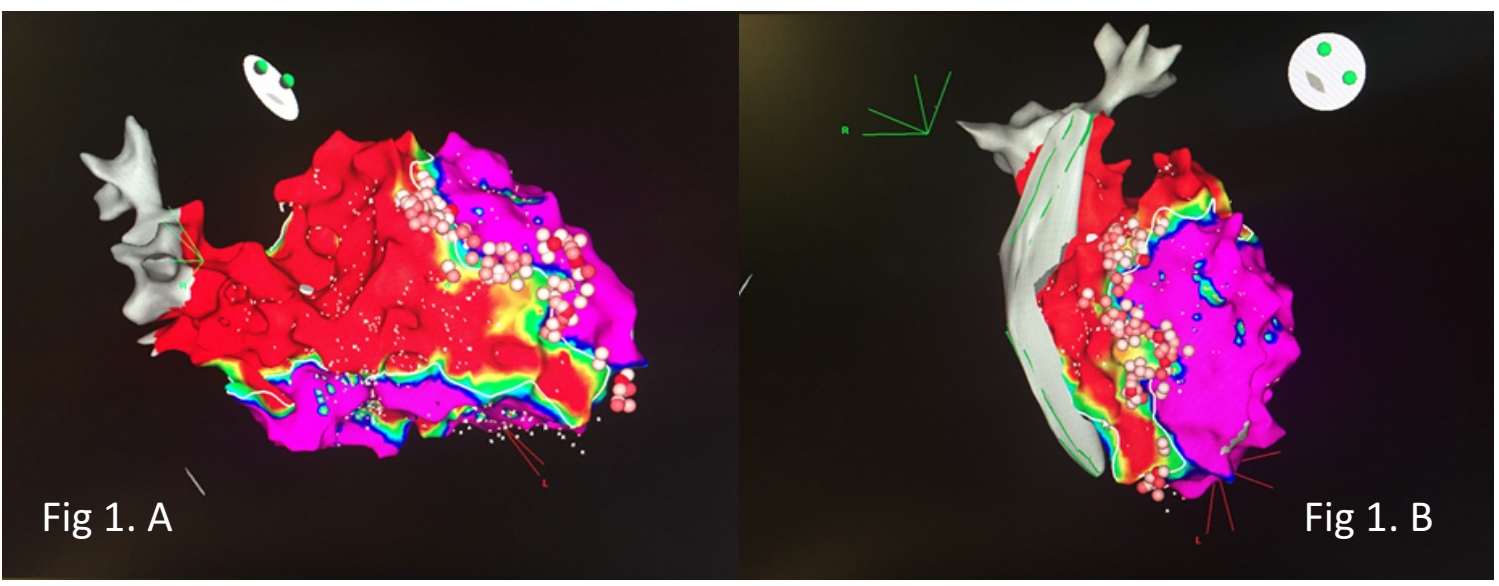

Figure 1: 3D endocardial electro anatomical voltage map of the left ventricle created at the time of catheter ablation of VT in Right Anterior Oblique (A) and a modified Antero-Posterior (B) views. The areas in red (antero-septal segment of the LV) indicated abnormal voltage suggesting substrate ("scar") for ventricular tachycardia. Areas in pink indicate normal voltage suggesting "healthy" myocardium. Areas in between red and pink indicate "scar boarder" where radiofrequency ablation (white/light pink circles) was performed.

On the other end of the spectrum, in patients with structural heart disease and advanced heart failure, where the incidence of recurrent $\mathrm{VA}$ is as high as $33 \%$, ES may be considered as a clinical marker or precursor of pump failure. Outcomes following ES in this situation is similar to those seen in patients presenting with decompensated failure without VA [12]. A recent review article by Santangeli [13] describes the common mechanisms involved in heart failure decompensation and VA (neuro-hormonal activation creating an imbalance between sympathetic and parasympathetic regulation). The whole process is a vicious cycle where advanced heart failure (due to various cardiomyopathies) leads to progressive myocardial remodeling/ scarring which sets up the substrate for recurrent VA which in turn contributes to further progression of heart failure leading to mortality. In these patients, traditional anti-arrhythmics have negative inotropic effects with the exception of quinidine/mexiletine (not very efficacious) and amiodarone (efficacious but serious side effects). This necessitates other therapeutic approaches such as catheter ablation (following optimization of clinical heart failure with medical therapy \pm ventricular assist device) and cardiac sympathetic modulation.

\section{Conclusion}

Our case report highlights the importance of a multi-disciplinary approach when managing complex patients with structural heart disease presenting with incessant/drug refractory VA. Growing evidence supports the participation of cardiac sympathetic system in these arrhythmias and serves as a target for successful treatment of these patients. Where anti-arrhythmic agents fail to suppress electrical storm, propofol has been repeatedly shown to eliminate VA in the acute setting by virtue of its effect on the cardiac sympathetic system. This can be used in the emergent setting to stabilize patients, serving as a bridge therapy and providing valuable time until more definitive therapies such as sympathetic modulation by ganglion block or surgical denervation can be safely performed.

\section{References}

1. Hohnloser S (2006) Electrical storm in patients with an implantable defibrillator: incidence, features, and preventive therapy: insights from a randomized trial. Eur Heart J 27: 3027-3032.

2. Pedersen C (2014) EHRA/HRS/APHRS Expert Consensus on Ventricular Arrhythmias. Heart Rhythm 11: 10.

3. Warpechowski P (2010) Effects of Propofol on the Cardiac Conduction System: Rev Bras Anestesiol 60: 438-444.

4. Mulpuru S (2008) Electrical storm and termination with propofol therapy. Int J Cardiol 128: e6-e8.

5. Burjorjee J (2002) Propofol for electrical storm; a case report of cardioversion and suppression of ventricular tachycardia by propofol. Can J Anesth 49: 973-977.

6. Liu Q (2011) Propofol and arrhythmias: two sides of the coin. Acta Pharmacologica Sinica 32: 817-823. 
Citation: Venkatesh K, Acevedo J, Santos MD, Kim N, Catanzaro J, et al. (2017) Management of Electrical Storm: The Answer Lies outside the Heart. Cardiovasc Pharm Open Access 6: 213. doi:10.4172/2329-6607.1000213

Page 3 of 3

7. Vaseghi M (2012) A sympathetic innervation of the anterior left ventricular wall by the right and left stellate ganglia. Heart Rhythm 9: 1303-1309.

8. Nademanee K (2010) Treating electrical storm: sympathetic blockade versus ACLS support-guided therapy. Circulation 102: 742-747.

9. Shivkumar K (2010) Neuraxial modulation for refractory ventricula arrhythmias: Value of thoracic epidural anesthesia and surgical left cardiac sympathetic denervation: Circulation 121: 2255-2262.

10. Grimaldi R (2012) Can spinal cord stimulation reduce ventricular arrhythmias. Heart Rhythm 9.
11. Vaseghi M (2014) Cardiac Sympathetic Denervation in patients with refractory ventricular arrhythmias or electrical storm; intermediate and long term follow up. Heart Rhythm 11.

12. Guerra F (2015) Electrical storm and heart failure worsening in implantable cardiac defibrillator patients. Europace 17: 247-254.

13. Santangeli P (2017) Management of ventricular arrhythmias in patients with advanced heart failure. JACC 69: 1842-1860. 